\title{
Use of clobetasol in lacquer for plaque psoriasis treatment ${ }^{*}$
}

\author{
Suze Aparecida da Silva ${ }^{1}$ \\ Rafael Augusto Tamasauskas Torres ${ }^{2}$ \\ Paulo Eduardo Neves Ferreira Velho ${ }^{1}$
}

\author{
Renata Ferreira Magalhães ${ }^{1}$ \\ Raquel Diana de Oliveira ${ }^{3}$
}

DOI: http:/ / dx.doi.org/10.1590/abd1806-4841.20164228

\begin{abstract}
Clobetasol benefits to control psoriasis lesions are well defined, but there were not studies about its action when used in lacquer vehicle to control skin lesions. A double-blind study was conducted with 40 patients that utilized clobetasol $0.05 \%$ in one hemibody and just the vehicle in the other hemibody. Twenty of them used petrolatum as vehicle and the others used lacquer. An assessment was conducted using the clinical index PASI and a quality of life questionnaire (Dermatological Life Quality Index). There was no statistical difference between groups. There was a trend of favorable response particularly in the hemibody treated with clobetasol.
\end{abstract}

Keywords: Clobetasol; Psoriasis; Therapeutics

Psoriasis is a chronic, immune-mediated and genetic based dermatoses, characterized by accelerated cell proliferation of keratinocytes and by the inflammation that originate parakerotosic scales. ${ }^{1,2}$ Its cause is still unknown. However, it is known that its onset or exacerbation depend on several factors: genetic, immunological, environmental, and biochemical, besides the influence of emotional factor. ${ }^{2,3}$

Treatment may be topical or systemic. Use of clobetasol propionate, a synthetic glucocorticosteroid with high power, is already known and widely used. ${ }^{1,4}$ Most patients (80\%) present mild clinical form of the disease and are candidates for topical treatment. Even patients with extensive forms of the disease often continue with resistant lesions after systemic treatment and require association with topic medications. Treatment is also problematic due to the fact that disease manifestations are not uniform and due to adhesion difficulties. ${ }^{5,6}$ In the case of topical treatments, it occurs mostly due to cosmeceutical issues (drugs that have unpleasant odor or that dirty the clothes). ${ }^{7,8}$ Therefore, we sought a vehicle option that could increase adherence to treatment. The use of clobetasol in lacquer still hadn't been evalu- ated in skin lesions and was expected to eliminate the mentioned cosmetic factors that occur with certain ointments.

This study, developed after receiving authorization from the Ethics Committee of the Medical School (FCM)/ Unicamp, was prospective and double-blind. We selected two groups of 20 patients. Both groups used clobetasol $0.05 \%$ : the first group used lacquer as vehicle and the second used petrolatum. In both cases, volunteers were given two bottles, one with " $R$ " indication, to be used on the right hemibody, and another with " $\mathrm{L}$ " indication, to be used on the left hemibody. Only one of the bottles contained the active ingredient and only the person responsible for the formulation knew this information.

For the selection of volunteer patients we used PASI index (Psoriasis Area and Severity Index), which assesses the extent and severity of psoriasis. With the assessment of PASI it is possible to classify psoriasis as mild, moderate and severe based on skin manifestations. The disease is considered mild when indexes are $<10$ and moderate to severe when they are $>10$. ${ }^{1,9}$ Patients assisted by the Dermatology Department of the State

Received on 19.11.2014

Approved by the Advisory Board and accepted for publication on 03.03.2015

Study performed at Faculdade de Ciências Médicas da Universidade Estadual de Campinas (Unicamp) - Campinas (SP), Brazil.

Financial Support: FAPESP: research support 2008/09433-9 CNPq: junior research grant.

Conflict of Interest: None.

Universidade Estadual de Campinas (Unicamp) - Campinas (SP), Brazil

Universidade de São Paulo - São Paulo (SP), Brazil

Private clinic - Campinas (SP), Brazil

(C2016 by Anais Brasileiros de Dermatologia 

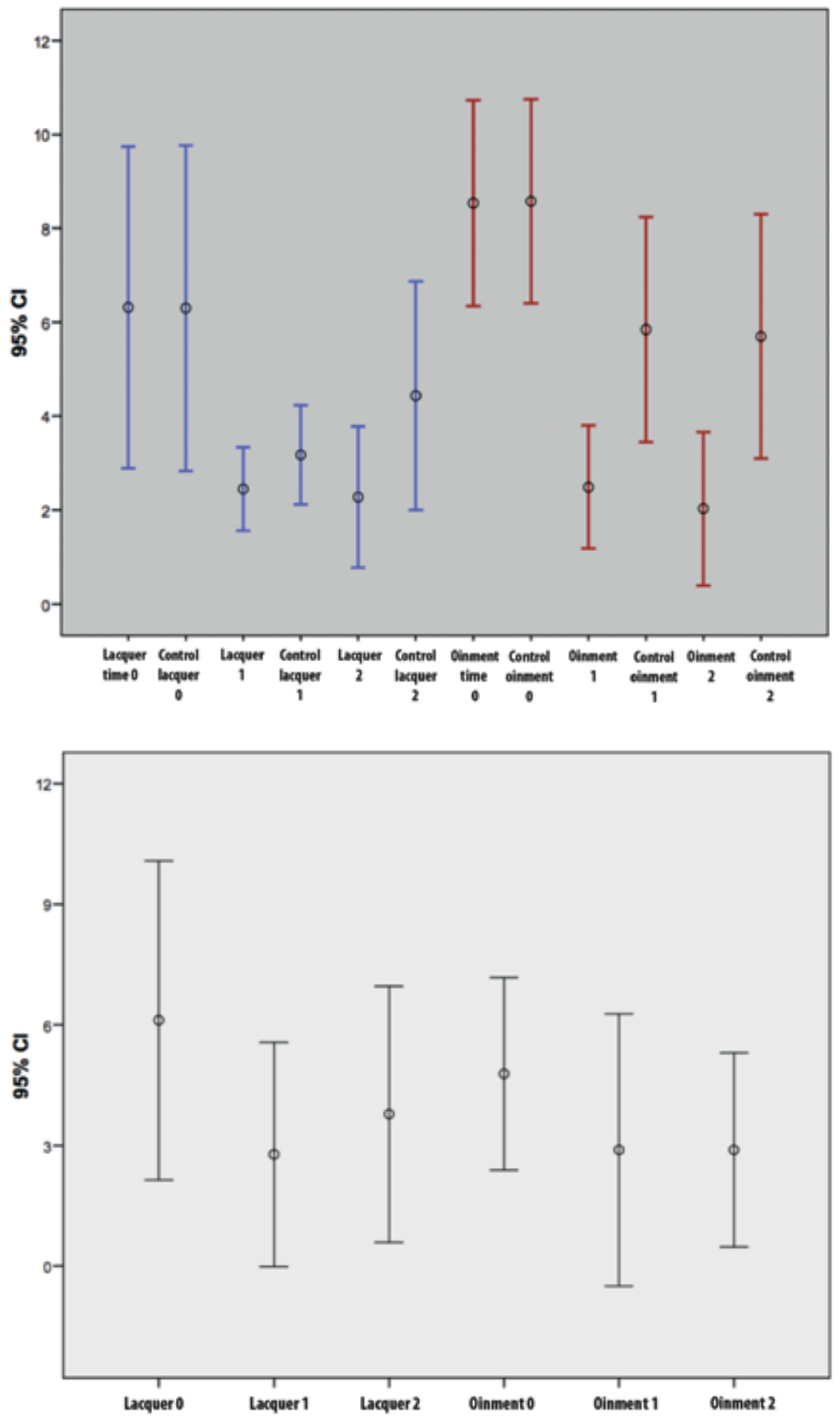

FIGURE 1: Distribution of PASI comparing the evolution of hemibodies exposed to clobetasol in lacquer, in ointment and its control
FIGURE 2: Distribution of DLQI comparing the outcomes of patients who used lacquer and those using the ointment
University of Campinas Medical School (FCM-UNICAMP), presenting mild to moderate psoriasis at the beginning of the protocol, participated in the research. The study selected individuals with a minimum age of 18 years, with no use of topical treatments or sudden changes of clinical presentation for at least two months. Patients with severe form of the disease at baseline or known allergy to lacquer were excluded.
The study included 40 patients, 24 men. Mean age was 53.9 years, ranging between 18 and 81 years.

In the initial consultation, after prior consent by volunteers, the attending physician determined the clinical index of each hemibody, based on PASI criteria. The same procedure was conducted in consultations in Day 30 and Day 60 of treatment. Patients also answered, in each of these consultations, to the 
self-administered questionnaire DLQI (Dermatological Life Quality Index) used to assess quality of life. ${ }^{10}$

In the end, clinical rates obtained were confronted with the information declared by the pharmaceutical and the analysis of the DLQI values was performed. For the comparative analysis of the results, we used the SPSS 15.0 program.

Three patients in each group did not complete the proposed two months of treatment. In the group using lacquer as vehicle, two patients reported severe pruritus associated with erythematous lesions after a few days of application of the lacquer, and another patient was withdrawn due to clinical worsening. Use of lacquer in extensor areas, such as knee and elbow, has also been criticized by two patients who completed the study, due to the occurrence of dryness, breaking of the film and and desintegration of the product. In the group using petrolatum as vehicle, two patients left the protocol and another presented allergy referred as eyelid swelling and pruritus on the face, which led to the suspension of topical use.

Clinical rates of each hemibody showed an improvement tendency in both groups of patients. This tendency was also observed even in hemibodies in which only the vehicle was applied, according to the graphics on the mean and standard deviation for a $95 \%$ confidence interval. Considering the mean, there was an improvement in both groups, most evident within the first month of treatment. The hemibodies exposed to lacquer showed a higher improvement tendency compared with that observed in control hemibodies. Observing the mean, this tendency was slightly higher in hemibodies treated with clobetasol ointment. As standard deviations touch, there was no statistically significant difference between groups (Figure 1). Tendency of improvement in the hemibody exposed only to vehicle may represent a placebo effect of topical use, but the possibility of absorption of the active ingredient in both vehicles and hydrating/moisturizing potential of the ointment vehicle should be considered.

Results obtained with DLQI are presented in figure 2, whose distribution shows that there was a tendency toward improvement of quality of life in both groups, with no statistical difference between them.

Results suggest that use of clobetasol in lacquer must be considered, especially in the most localized lesions and psoriasis thinner plaques, besides the use in nail lesions.

\section{ACKNOWLEDGEMENTS:}

The authors thank the pharmacist Eduardo Aldrin Marcos, owner of the Botica Erva Doce, where the products were manufactured.

\section{REFERENCES}

1. Sociedade Brasileira de Dermatologia. Consenso Brasileiro de Psoríase 2009. Rio de Janeiro: Sociedade Brasileira de Dermatologia; 2009.

2. Perera GK, Di Meglio P, Nestle F0. Psoriasis. Annu Rev Pathol. 2012;7:385-422.

3. Sociedade Brasileira de Dermatologia. Consenso Brasileiro de Psoríase 2012: Guias de Avaliação e Tratamento. 2. ed. Rio de Janeiro: Sociedade Brasileira de Dermatologia; 2012.

4. Nakamura RC, Abreu Ld, Duque-Estrada B, Tamler C, Leverone AP. Comparison of nail lacquer clobetasol efficacy at $0.05 \%, 1 \%$ and $8 \%$ in nail psoriasis treatment: prospective, controlled and randomized pilot study. An Bras Dermatol. 2012;87:203-11.

5. Zaghloul SS, Goodfield MJ. Objective assessment of compliance with psoriasis treatment. Arch Dermatol. 2004;140:408-14.

6. Richards HL, Fortune DG, O'Sullivan TM, Main CJ, Griffiths CE. Patients with psoriasis and their compliance with medication. J Am Acad Dermatol. 1999;41:581-3

7. Devaux S, Castela A, Archier E, Gallini A, Joly P, Misery L, et al.. Adherence to topical treatment in psoriasis: a systematic literature review. J Eur Acad Dermatol Venereol. 2012;26:61-7.

8. Bewley A, Page B. Maximizing patient adherence for optimal outcomes in psoriasis J Eur Acad Dermatol Venereol. 2011;25:9-14.

9. Torres RA, Silva SA, Magalhães RF, Morcillo AM, Velho PE. Comparison of quality of life questionnaires and their correlation with the clinical course of patients with psoriasis. An Bras Dermatol. 2011;86:45-9.

10. Martins GA, Arruda L, Mugnaini ASB. Validation of life quality questionnaires for psoriasis patients. An Bras Dermatol. 2004;79:521-35.
MAILING ADDRESS:

Paulo Eduardo Neves Ferreira Velho

Rua: Tessália Vieira de Camargo, 126

Cidade Universitária "Zeferino Vaz"

13083-887 Campinas, SP.

Email:pvelho@unicamp.br

How to cite this article: Silva AS, Magalhães RF, Torres RAT, Oliveira RD, Velho PENF. Use of clobetasol in lacquer for plaque psoriasis treatment. An Bras Dermatol. 2016:91(1):113-5. 